\title{
Integrated Simulation and Optimization Scheme of Real-time Large Scale Water Supply Network: Applied to Catalunya Case Study
}

\author{
Cong Cong Sun ${ }^{\mathrm{a}}$, Vicenç Puig ${ }^{\mathrm{a}, *}$, Gabriela Cembrano ${ }^{\mathrm{a}, \mathrm{b}}$ \\ ${ }^{a}$ Advanced Control Systems Group at the Institut de Robòtica i Informàtica Industrial \\ (CSIC-UPC), Llorens i Artigas, 4-6, 08028 Barcelona, (Spain). Tel: +34 688041855 \\ ${ }^{b}$ CETaqua, Water Technology Centre at Esplugues 75, 08940, Cornellà de Llobregat, Barcelona
}

\begin{abstract}
This paper presents an integrated simulation and optimization modelling approach in order to provide the optimal configuration for the large scale water supply system (LSWSS) in real time. Model Predictive Control (MPC) has been chosen to handle the complex set of objectives and also being shown its effectiveness. The computation of control strategies by MPC uses a simplified model of the network dynamics. The use of the combined approach of optimization and simulation contributes to making sure that the effect of more complex dynamics, better represented by the simulation model, may be taken into account. Coordination between simulator and optimizer works in a feedback scheme, from which both real-time interaction and also extensive validation of the proposed solution have been realized by realistic demonstrations. The Catalunya regional water network has been used as the case study.
\end{abstract}

*Corresponding author

Email address: vicenc . puig@upc . edu (Vicenç Puig)

The manuscript of this paper has been received on 2nd, February, revised on 06th, June and accepted on 20th of October, 2014. 
Keywords: MPC, simulation, optimization, coordination

\section{Introduction}

Impacts of climate changes are likely to give rise to uncertainties in water availability and water demands, which may results in major economical and ecological consequences. [1] presented a discussion of uncertainty paradigms in water resources, and provided his views on water management tools that can be used in the future.

LSWSS, which is composed of various physical elements such as reservoirs, channels, pumping stations, irrigation area and urban water supply systems, operates to supply water for municipal, industrial and irrigation needs. Management of these systems from planning to operation is very challenging since the problem deals with many complex modelling issues related to inflows, transportation delays, storage, irrigation and industrial as described at [2]. An effective management of LSWSS requires a supervisory control system that takes optimal decisions about the current operational configuration of the whole network. Such decisions are implemented automatically or offered as a decision support to operators and managers. The control system should take into account operating constraints, costs and consumer demands. The decisions of the control systems are translated into set-points to individual, localized, lower level systems that optimize the pressure profile to minimize losses by leakage and provide sufficient pressure. The whole control system responds to changes in network topology (ruptures), typical daily/weekly profiles, as well as major changes in demand as discussed in [3].

A number of systems analysis techniques involving simulation and optimization algorithms have been developed and applied over the last several decades 
to study LSWSS and also have been reviewed in [4], [5] and [6]. [4] provides a comprehensive state-of-the-art review of theories and applications of systems analysis techniques to LSWSS with a strong emphasis on optimization methods. LSWSS simulation and optimization models were reviewed by [5] who evaluated the usefulness of each approach for different decision support situations in order to provide better understanding of modelling tools which could help the practitioner in choosing the appropriate model. [6], in his review on optimal operation of LSWSS, suggested the need to improve operational effectiveness and efficiency of water resources systems through the use of computer modelling tools. Continuous development in information technology (hardware and software) creates a good environment for transition to new decision making tools. Spatial decision support systems using object oriented programming algorithms are integrating transparent tools that will be easy to use and understand at [1]. A number of text books on modelling and systems analysis of water resources including LSWSS are available like [2], [7], [8], [9], [10], [11] and [12].

MPC has been proven to be one of the most effective and accepted control strategies for the global optimal operational control of large-scale water networks in [13]. Applications to different large-scale infrastructures as LSWSS as in [14], sewer networks in [15], open-flow channel networks at [16] or electrical networks in [17] prove the applicability of this technique. The main characteristic is that after the plant dynamical model has been obtained, the MPC design just consists of expressing the desired performance specifications through different control objectives (e.g., weights on tracking errors and actuator efforts as in classical linear quadratic regulation), and constraints on system variables (e.g., minima/maxima of selected process variables and/or their rates of change) which are necessary to 
ensure process safety and asset health.

SIMULINK, as talked about in [18], is an environment for multi-domain simulation and model-based design for dynamic and control systems. It provides an interactive graphical environment and a customizable set of block libraries that allow to design, simulate, implement, and test a variety of systems, used in communications, control, signal processing, video processing, and image processing. According to these properties, SIMULINK is appropriate to develop a water network simulation environment that allows to include a network model and the cost function computation. This model allows to interface the controller, developed in this work in MATLAB using the MPC method, which provides the set points of the related elements and meanwhile close a control in a feedback loop as in [19].

The aim of this article is presenting simulation, optimization and integrated simulation-optimization modelling approaches in order to provide the optimal configuration for LSWSS in real time. MPC has been chosen to handle the complex set of objectives and also being shown its effectiveness. The computation of control strategies by MPC uses a simplified model of the network dynamics. The use of the combined approach of optimization and simulation contributes to making sure that the effect of more complex dynamics, better represented by the simulation model, may be taken into account. Coordination between simulator and optimizer works in an feedback scheme. The remainder of the paper is organized as follows. Control-oriented modelling methodology of MPC optimizer is first provided in Section 2. Then, in Section 3, controlling problem statement is described. In Section 4, integrated simulation and optimization scheme is explained in detail. Case study and preliminary results are outlined at Section 5 and finally, conclusion is provided at Section 6. 


\section{Control-Oriented Modelling Methodology}

Several modelling techniques dealing with the operational control of LSWSS have been presented in the literature, see [14], [20] and [21] and the references therein. Here, a control-oriented modelling approach is outlined, which follows the principles presented in the reference by [22] and [23]. The extension to include the pressure-model can be found in the references provided by [14] and [20]. A LSWSS generally contains tanks, which store the drinking water that comes from the network sources, a network of pressurized pipes and a number of sinks. Valves and/or pumping stations are elements that allow to manipulate the water flow according to a specific policy and to supply water requested by the network users.

\subsection{Tanks and Reservoirs}

Water tanks/reservoirs provide the entire network with the water storage capacity. The mass balance expression relating the stored volume $v$, the manipulated inflows $q_{\text {in }}^{i, j}$ and outflows $q_{\text {out }}^{i, l}$ (including the demand flows as outflows) for the $i$-th tank can be written as the discrete-time difference equation

$$
v_{i}(k+1)=v_{i}(k)+\Delta t\left(\sum_{j} q_{\text {in }}^{i, j}(k)-\sum_{l} q_{\text {out }}^{i, l}(k)\right) \text {, }
$$

where $\Delta t$ is the sampling time and $k$ denotes the discrete-time instant. The physical constraint related to the range of admissible water in the $i$-th tank is expressed as

$$
\underline{v}_{i} \leq v_{i}(k) \leq \bar{v}_{i}, \quad \text { for all } k,
$$

where $\underline{v}_{i}$ and $\bar{v}_{i}$ denote the minimum and the maximum admissible storage capacity, respectively. Although $\underline{v}_{i}$ might correspond to an empty tank, in practice 
this value can be set as nonzero in order to maintain an emergency stored volume enough to supply for facing extreme circumstances.

For simplicity purposes, the dynamic behavior of these elements is described as a function of volume. However, in most of the cases, the measured variable is the tank water level (by using level sensors), which implies the computation of the water volume taking into account the tank geometry.

\subsection{Actuators}

Two types of control actuators are considered: valves/gates and pumps (more precisely, pumping stations). The manipulated flows through the actuators represent the control variables, denoted as $q_{u}$. Both pumps and valves/gates have lower and upper physical limits, which are taken into account as system constraints. As in (2), they are expressed as

$$
{\underline{q_{u}}}_{i} \leq q_{u i}(k) \leq \overline{q_{u}}, \quad \text { for all } k,
$$

where ${\underline{q_{u}}}_{i}$ and $\overline{q_{u}}$ denote the minimum and the maximum flow capacity, respectively.

\subsection{Nodes}

These elements correspond to the points in the whole water system where water flows are merged or split. Thus, the nodes represent mass balance relations, being modelled as equality constraints related to inflows (from other tanks through valves or pumps) and outflows, the latter being represented not only by manipulated flows but also by demand flows. The expression of the mass conservation in these nodes can be written as

$$
\sum_{j} q_{\text {in }}^{i, j}(k)=\sum_{h} q_{\text {out }}^{i, h}(k) .
$$


Node inflows and outflows are still denoted by $q_{\text {in }}$ and $q_{\text {out }}$, respectively, despite the fact that they can be manipulated flows and hence denoted by $q_{u}$, if required.

\subsection{River Reaches}

A single canal reach can be approximated by using the modelling approach proposed by [24] that leads to the following relation between the upstream $\left(q_{\text {ups }}\right)$ and downstream $\left(q_{d n s}\right)$ flows:

$$
q_{d n s}(k+1)=a_{1} q_{d n s}(k)+b_{0} q_{u p s}(k-d)
$$

where $d=\tau_{d} / T_{s}, \tau_{d}$ is the downstream transport delay, $T_{s}$ is the sampling time, $b_{0}=1-a_{1}$ and $a_{1}=e^{-\frac{T_{s}}{T}}$.

\subsection{Demand and Irrigation Sectors}

Demand and irrigation sectors represent the water demand made by the network users of a certain physical area. For the purpose of computing control actions, it is considered as a measured disturbance of the system at a given time instant. The demand in urban areas can be anticipated by a forecasting algorithm that is integrated within the MPC closed-loop architecture in [13]. The demand forecasting algorithm typically uses a two-level scheme composed by $(i)$ a timeseries model to represent the daily aggregate flow values, and (ii) a set of different daily flow demand patterns according to the day type to cater for different consumption during the weekends and holidays periods. Every pattern consists of 24 hourly values for each daily pattern as explained in [25]. This algorithm runs in parallel with the MPC algorithm. The daily series of hourly-flow predictions are computed as a product of the daily aggregate flow value and the appropriate 
hourly demand pattern. On the other hand, irrigation demand is typically planned in advance with farmers. Pre-established flows for irrigation are planned for agricultural areas in certain periods of the year.

\section{Control Problem Statement}

Because of the complex large scale property and multi-variable control characteristic of LSWSS as described in Section 2, a feasible and efficient control method is needed.

MPC is one of the most advanced control methodologies which has made a significant impact on industrial control. MPC does not consider a specific control strategy but a very wide range of control methods which make an explicit use of the process model to obtain the control signal by minimizing an objective function which represents the desired control goals. MPC can handle multi-variable control problems and it can consider actuator limitations as well as operational and physical constraints.

The standard MPC problem based on the linear discrete-time prediction model is considered as described in [26]:

$$
\begin{aligned}
x(k+1) & =A x(k)+B u(k), \\
y(k) & =C x(k),
\end{aligned}
$$

where $x(k) \in \mathbb{R}^{n_{x}}$ is the state vector and $u(k) \in \mathbb{R}^{n_{u}}$ is the vector of command variables at time step $k$, and $y(k) \in \mathbb{R}^{n_{y}}$ is the vector of the measured outputs. Following the formalism provided by [26] for the basic formulation of a predictive control, the cost function is assumed to be quadratic and the constraints are in the form of linear inequalities. The model (6) is obtained applying the control- 
oriented modelling methodology presented in previous section taking into account the LSWSS topology and physical parameters.

\subsection{Operational Goals}

The main operational goals need to be achieved in LSWSS are:

- Goal 1. Cost reduction $\left(J_{\text {cost }}\right)$ : Economic cost is usually includes water source costs and water transportation costs two parts. Water source cost is usually related to acquisition, which may have different prices at different sources, while transportation cost is affected by power tariffs which may vary during different time steps in a day.

Assuming $W_{a}$ as the related weight of Goal 1 , the vectors $\widetilde{u}, a_{1}$ and $a_{2}$ contain control variables, cost of water source and pumping at time step $k$, respectively, the object function of Goal 1 is

$$
J_{\text {cost }}=W_{a}\left(a_{1}+a_{2}(k)\right) \widetilde{u}(k),
$$

- Goal 2. Operational safety $\left(J_{\text {safety }}\right)$ : This criterion refers to maintain appropriate water storage levels in dams and reservoirs for emergency-handling. Operated in both supply and transportation layers.

Assuming $W_{\widetilde{x}}$ as the related weight of Goal 2, $\widetilde{x}(k)$ and $\widetilde{x}_{r}$ represent realtime water level and emergency-handling level in dams and reservoirs at time step $k$, respectively, the object function of Goal 2 is

$$
J_{\text {safety }}=\left(\widetilde{x}(k)-\widetilde{x}_{r}\right)^{\top} W_{\widetilde{x}}\left(\widetilde{x}(k)-\widetilde{x}_{r}\right),
$$


- Goal 3. Balance management ( $\left.J_{\text {balance }}\right)$ : This is operated only at supply layer which is necessary for keeping rivers or reservoirs consumed in a balanced way and escaping water deficit problem for both of the two rivers in the long run.

Assuming $w_{\widetilde{m}}$ as the related weight of Goal 3, $x_{i}$ and $x_{j}$ are two main reservoirs located in two different rivers, the object function of Goal 3 is

$$
\begin{aligned}
J_{\text {balance }} & =\left(\left(\begin{array}{llllllll}
0 & \ldots & \frac{1}{x i_{\max }^{\prime}} & 0 \ldots 0 & \frac{-1}{x j_{\max }^{\prime}} & 0 \ldots 0
\end{array}\right) \widetilde{x}(k)\right)^{\top} w_{\widetilde{m}} \\
& \times\left(\left(\begin{array}{llllllll}
0 & \ldots & \frac{1}{x i_{\max }^{\prime}} & 0 \ldots 0 & \frac{-1}{x j_{\max }^{\prime}} & 0 \ldots 0
\end{array}\right) \widetilde{x}(k)\right)
\end{aligned}
$$

- Goal 4. Demand management $\left(J_{\text {demand }}\right)$ : This is especially important in the supply layer when urban and irrigation demands exist since irrigation demands allow some degree of slackness.

Assuming $w_{\widetilde{f}}$ as the related weight of Goal 4, $\varepsilon(k)$ is vector of irrigation demand slackness, the object function of Goal 4 is

$$
J_{\text {demand }}=\varepsilon(k)^{\top} W_{f} \varepsilon(k),
$$

- Goal 5. Minimizing waste $\left(J_{\text {mwaste }}\right)$ : To take into account that the river water eventually goes to the sea, this term gets to avoid unnecessary water release from reservoirs (releasing water that does not meet any demand and is eventually wasted).

Assuming $w_{\widetilde{w}}$ as the related weight of Goal 5, variables $\widetilde{u}_{i \ldots j}(k)$ are the flows from the rivers to the sea. $\widetilde{u}_{s}(k)$ are their ecological penalty levels, the object function of Goal 5 is 


$$
\left.J_{\text {mwaste }}=\left(\widetilde{u}_{i \ldots j}(k)-\widetilde{u}_{s}(k)\right)^{\top} W_{\widetilde{w}} \widetilde{u}_{i \ldots j}(k)-\widetilde{u}_{s}(k)\right),
$$

- Goal 6. Environment conservation ( $J_{\text {ecological }}$ ): Water sources such as boreholes, reservoirs and rivers are usually subject to operational constraints to maintain water levels and ecological flows. The objective function of Goal 6 is contained in (8) because that water flows in the rivers are modelled as additional state variables as discussed before.

Above mentioned goals lead to the following optimized function:

$$
\begin{aligned}
J & =J_{\text {safety }}+J_{\text {demand }}+J_{\text {mwaste }}+J_{\text {balance }}+J_{\text {cost }} \\
& =\varepsilon_{\widetilde{x}}(k)^{\top} W_{\widetilde{x}} \varepsilon_{\widetilde{x}}(k)+\varepsilon(k)^{\top} W_{f} \varepsilon(k) \\
& +\left(\widetilde{u}_{i \ldots j}(k)-\widetilde{u}_{s}(k)\right)^{\top} W_{\widetilde{w}}\left(\widetilde{u}_{i \ldots j}(k)-\widetilde{u}_{s}(k)\right) \\
& +\left(\left(\begin{array}{lllllll}
0 & \ldots & \frac{1}{x i_{\text {max }}^{\prime}} & 0 \ldots 0 & \frac{-1}{x \dot{j}_{\max }} & 0 \ldots & 0
\end{array}\right) \widetilde{x}(k)\right)^{\top} w_{\widetilde{m}} \\
& \times\left(\left(\begin{array}{llllll}
0 & \ldots & \frac{1}{x i_{\max }^{\prime}} & 0 \ldots 0 & \frac{-1}{x \dot{j}_{\max }^{\prime}} & 0 \ldots
\end{array}\right) \widetilde{x}(k)\right) \\
& +W_{a}\left(a_{1}+a_{2}(k)\right) \widetilde{u}(k)
\end{aligned}
$$

where

$$
\varepsilon_{\bar{x}}(k)=\widetilde{x}(k)-\widetilde{x}_{r}
$$

The weight tuning method proposed in [27], based on computing the Pareto front of the multi-objective optimization problem presented in (13), is used in this paper. The initial step of this tuning approach is to find what are known as the 
anchor points that correspond to the best possible value for each objective obtained by optimizing a single criterion at a time. Then, a normalization procedure is applied, a Management Point (MP) defined by establishing objective priorities is defined, and the optimal weights are determined by computing those that minimize the distance from the solutions of the Pareto front and the MP.

\section{Integrated Simulation and Optimization Scheme}

Simulation could be the starting point in the planning of LSWSS but in view of the large number of configuration options, capacity and operating policy, simulation without preliminary screening through optimization would be very time consuming. The studies of large scale systems at [28] and [29] have indicated that even with the use of simple programming approaches such as LP, valuable improvement can be obtained to organize simulation.

Identifying effective pre-defined operating rules for simulating complex water supply systems is a challenging task. To overcome this problem the researchers generally employ optimization methods to simulation models like [29] and [30].

\subsection{General Structure}

In LSWSS, simulation and optimization are integrated in the feedback way as provided in Fig. 1. It shows that, simulation and MPC optimization models are working interactively by communicating mutual information. In every iteration, the MPC optimizer provides optimized control actions as set-point flows to the simulator. After being simulated, the produced state variables, which represent tanks/reservoirs volume, are sent back to the MPC optimization model as initial tanks/reservoirs volume for the next iteration. 


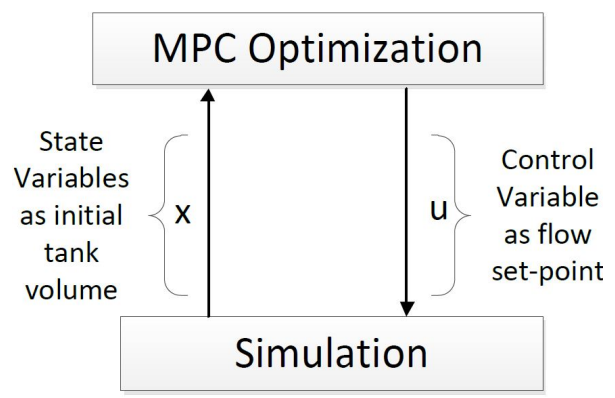

Figure 1: Feedback structure of Simulation and Optimization

\subsection{Simulation}

In spite of the development of optimization methodologies, simulation modelling techniques remain in practice a prominent tool for LSWSS planning and management studies. Simulators associated with LSWSS are usually based on mass balance equations and dynamic behavior of reservoir systems using inflows and other operating conditions. Application of simulation techniques to LSWSS planning and management started with U.S. Army Corps of Engineers (USACE), who built simulations of Missouri River. The famous Harvard Water Program applied simulation techniques to the economic design of water resources as shown in [31]. The simulated models produced the behavior for power generation, irrigation and flood control as reported in [2].

At the beginning, the simulator requires the parameters of every elements and the values of the actuator set-point or the demands as explained in [19]. All these data, are loaded from the database to the workspace, which has been saved in a different structure for each different element. When the simulator is connected directly to a controller developed in MATLAB, otherwise, the values of the simulation results have already been in the workspace, and it is sufficient to insert them in the correct structures. 


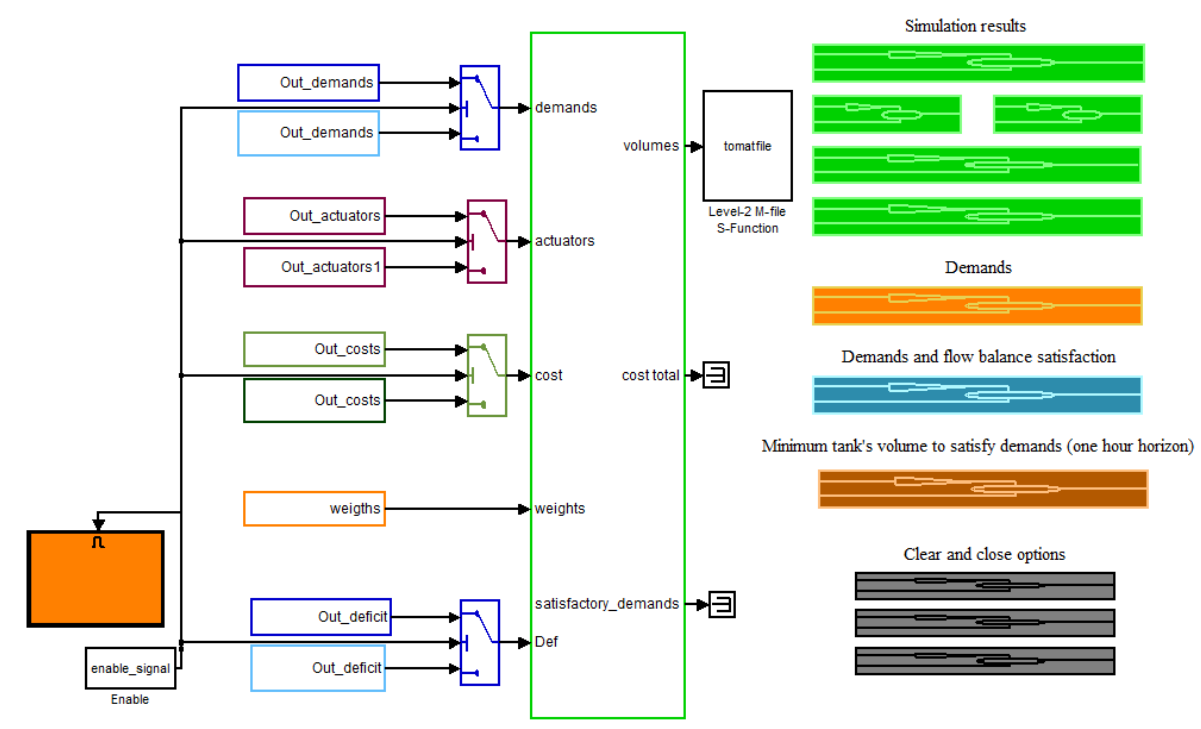

Figure 2: Main window of simulator

Fig. 2 is the main window of the LSWSS simulator environment, which includes inputs, outputs and also all the functional blocks needed during the whole simulating process. The blocks at the left side are the main inputs, providing and updating the required parameters (e.g. water demands, objective weights or electricity price of pumps) to the simulator by loading related data file. Blocks on the right side are the main outputs for visualizing the simulating results. Inside the center part, embedded the complete water network, see Fig. 4, which is the simulating of the regional water network of Catalunya case study as an example (see Section 5 for more detail).

\subsection{Optimization}

The MPC optimizer of LSWSS is presented in this section. The controller computes the optimal solution with a predictive horizon and a multi-objective cost function, which reflects the control strategy of water networks. At any time inter- 
val, only the first set-point value is used and at the next time interval a new computation is started. The results are obtained interfacing the simulator described in the section above, with the MATLAB platform with the help of TOMLAB/CPLEX optimizer.

\subsubsection{Formulation of the optimization problem}

The objective function (13) of the MPC problem can be formulated in the following way:

$$
J=z^{T} \Phi z+\phi^{T} z+c
$$

where

$$
z=\left[\begin{array}{lll}
\Delta \widetilde{u} & \varepsilon_{\widetilde{x}} & \varepsilon
\end{array}\right]^{T}
$$

$$
\Delta \widetilde{u}(k)=\widetilde{u}(k)-\widetilde{u}(k-1)
$$

and $c$ is a constant value produced by vector calculation.

This allows to determine the optimal control actions at each instant $k$ by solving a quadratic optimization problem by means of quadratic programming (QP) algorithm of the form:

$$
\begin{gathered}
\min _{z} z^{\top} \Phi x+\phi^{\top} z \\
A_{1} z \leq b_{1} \\
A_{2} z=b_{2}
\end{gathered}
$$

\subsection{Integration scheme of Simulator and Controller}

As described in Fig. 1 at the section above, the MPC controller coordinates with the simulator by communicating and exchanging mutual information. This 
integration is achieved by the two S-functions (see SIMULINK manual for more details) $S$-controller and $S$ - simulator, where they produce, transfer and receive the useful information in a closed loop as shown in Fig. 3. In this closed loop, the optimizer will first produce the optimized control actions and send them to the simulator as set-points. After the simulation, the updated states and the implemented control actions are transferred into the controller as state estimation and initial set-point values respectively for the next optimizing process. Initial data for the first optimizer process is provided. The scheme is working emulating real time operation by receiving and updating the demand and the measurements of the network real state from the telemetry system provided by SCADA system.

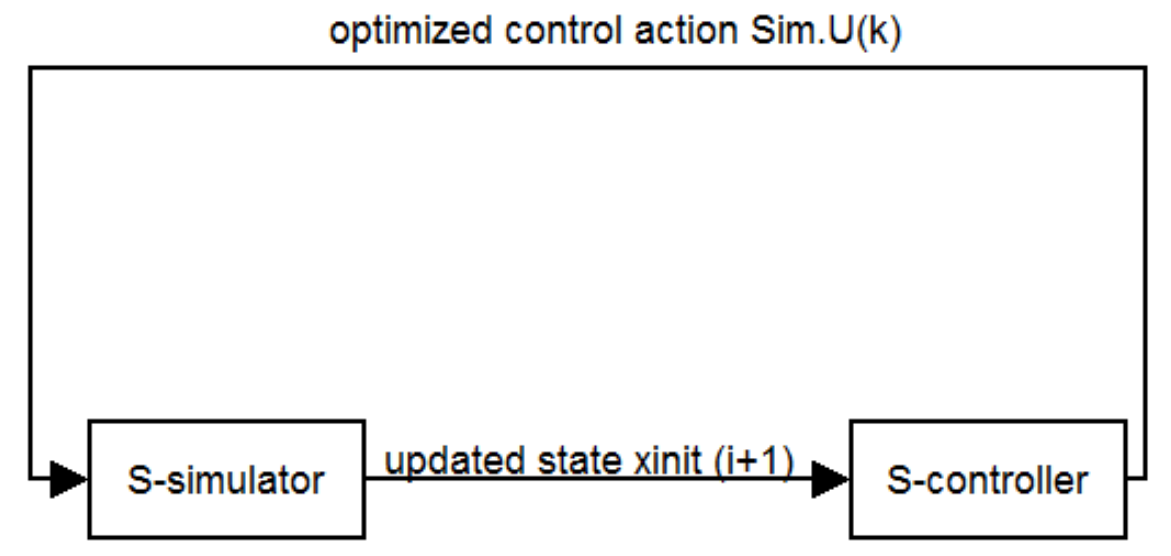

Figure 3: Integration of optimization and simulation blocks

In order to make sure the optimizer and simulator in the integrated scheme can work at a consistent pace, variable sampling steps have been used.

There are two sampling time deltaT and mindeltaT, where deltaT is the sampling time for MPC optimizer, which equals with 10800 seconds, while mindeltaT is the sampling time for simulator, here equals with 30 seconds. The two different 
sampling time synchronize the simulator and optimizer as presented in Algorithm 1 and are selected according to the network dynamics.

\section{Case Study: The Catalunya Regional Water Network}

\subsection{Description}

The Catalunya Regional Water Network lies within the Catalunya Inland Basins, and supplies the Metropolitan area of Barcelona, where most of the population is concentrated (approximately 5.5 million people). The sources of the Catalunya Regional Water Network are related mainly to two rivers (Llobregat and Ter). An assessment based on data obtained by the supply companies in the Barcelona metropolitan area shows that in 2007, 81 percent of the water input came from surface sources. Of the total water input, $90 \mathrm{hm}^{3}$ came from the Llobregat system and $124 \mathrm{hm}^{3}$ from the Ter system. The water flow supplied by the Ter and Llobregat rivers are regulated respectively by three and two reservoirs and purified by one and two water treatment plants, respectively.

Fig. 4 is the whole simulation network scheme of Catalunya Regional case study, where the two part at the sides are two rivers which names Llobregat and Ter and the center part is the aggregated network of water transportation in Barcelona city.

\subsection{Results}

Among demands in this network, some demands can receive water from both of the two rivers. In practical applications, there are three kinds of scenarios divided by the amount of water in Llobregat and Ter rivers, they are:

- Scenarios 1: More water in Llobregat than in Ter. 


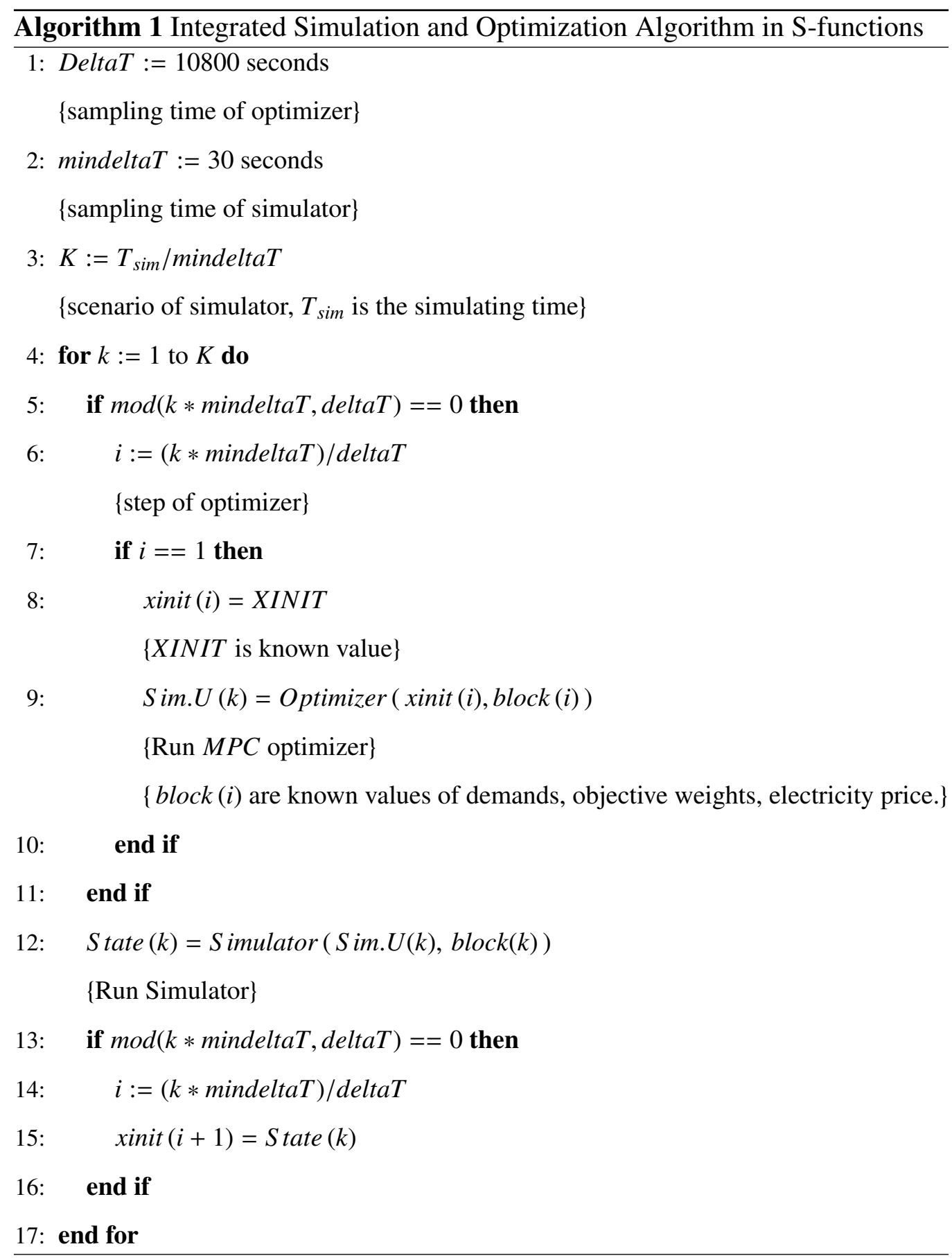




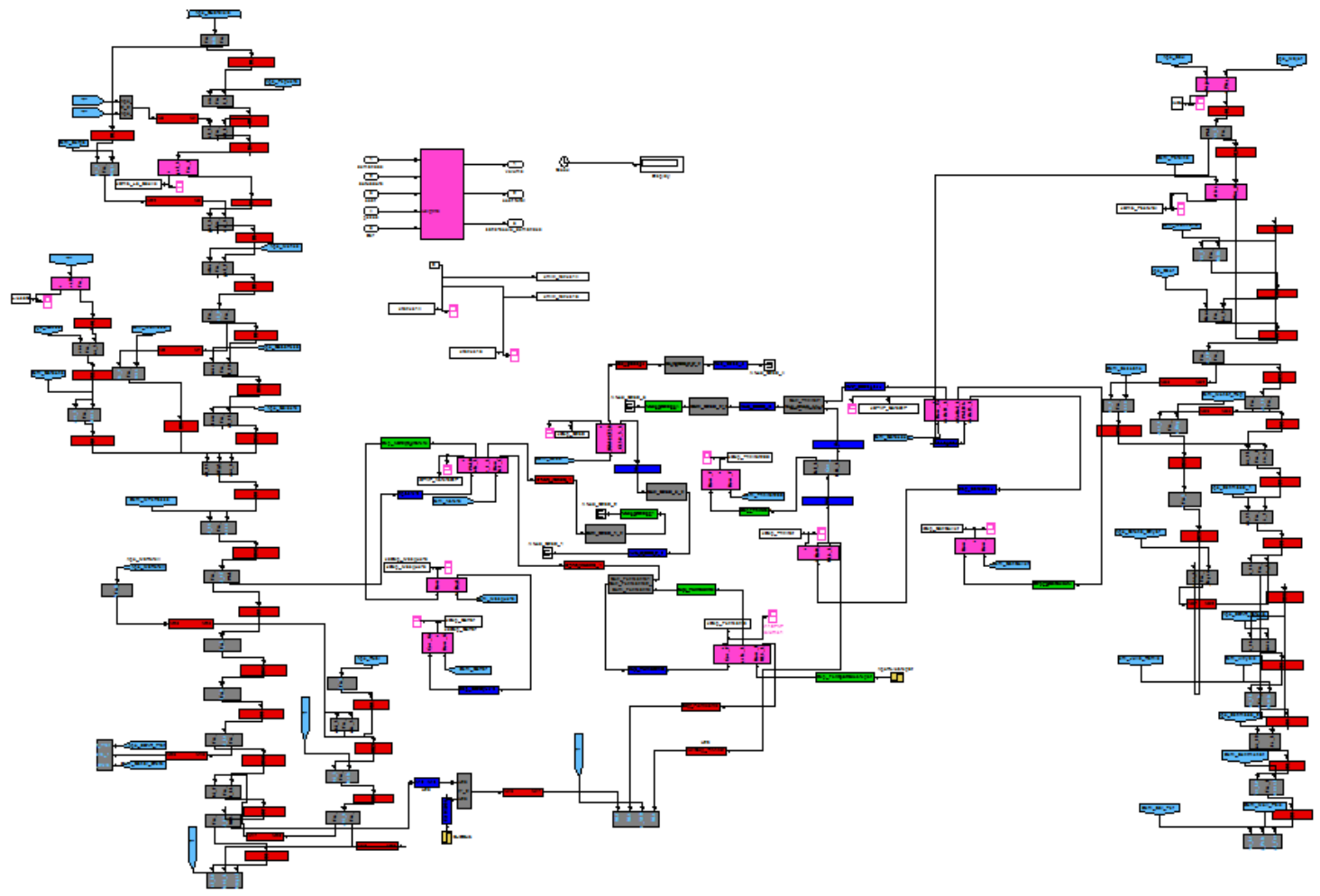

Figure 4: Simulation network scheme of Catalunya Regional Case Study 
- Scenarios 2: More water in Ter than in Llobregat.

- Scenarios 3: Abundant water in both of the rivers.

For the first two scenarios, when water in one river is adequate while in another river is limited, management policies will set the shared demands ask water from the abundant river. For the scenario 3, when water is abundant in both of rivers, according to the balance management, which is one of control objectives in the MPC controller, water consumption in both of rivers will be proportional to their supplying ability. In this paper, only scenario 3 is considered as the case study.

The following results are used to show the usefulness of this tool and also the benefits of the integrated scheme that make the water supply and transport keep the supply of both rivers balanced. In Section 5.2.1, optimal results of the MPC controller are provided while integrated results between MPC controller and simulator are described in Section 5.2.2.

\subsubsection{Results of Optimization}

In the Catalunya Regional Water Network, water transportation implies economic cost when pumped from the lower elevation to the higher elevation. As Goal 1, the MPC controller optimized this cost to produce the most optimal solution. Figure 5 shows a comparison between pump flow and its electricity fee where the pump works more during the cheaper time while it nearly does not work when the electricity price is high.

Fig. 6 shows water level of one reservoir comparing with its safety level, where the required storage is kept for emergency handling, which corresponds to the Goal 2.

Table 1 provides detailed results and also improvement of water usages in the 


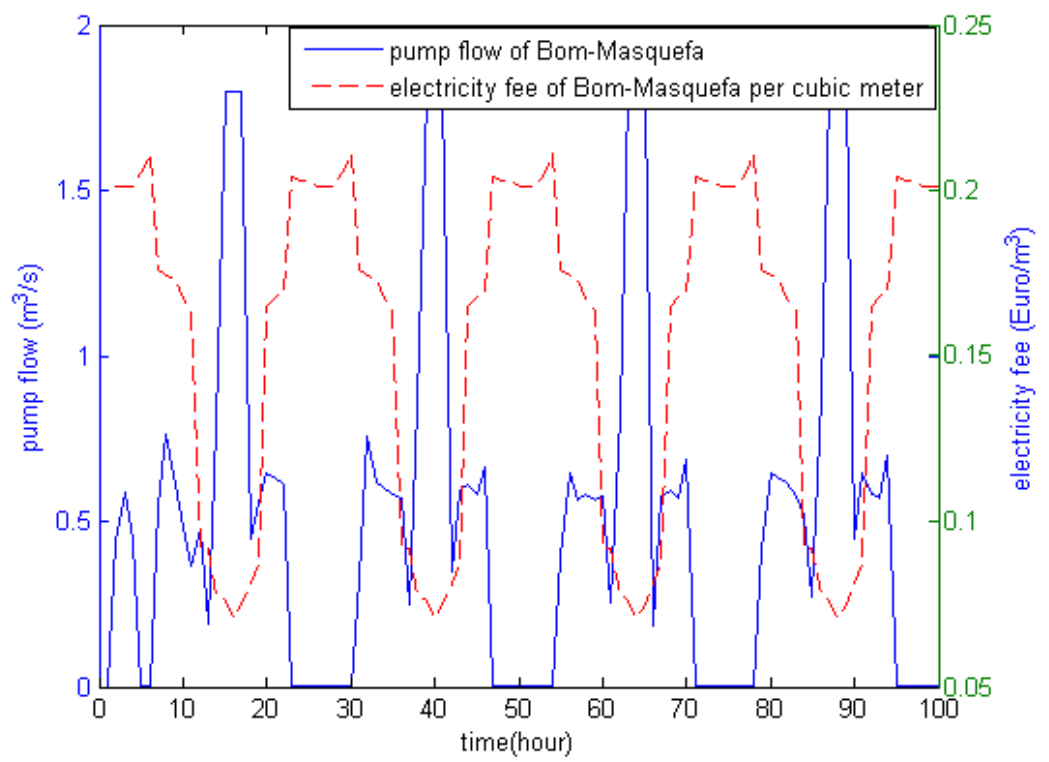

Figure 5: Pump flow with electricity price

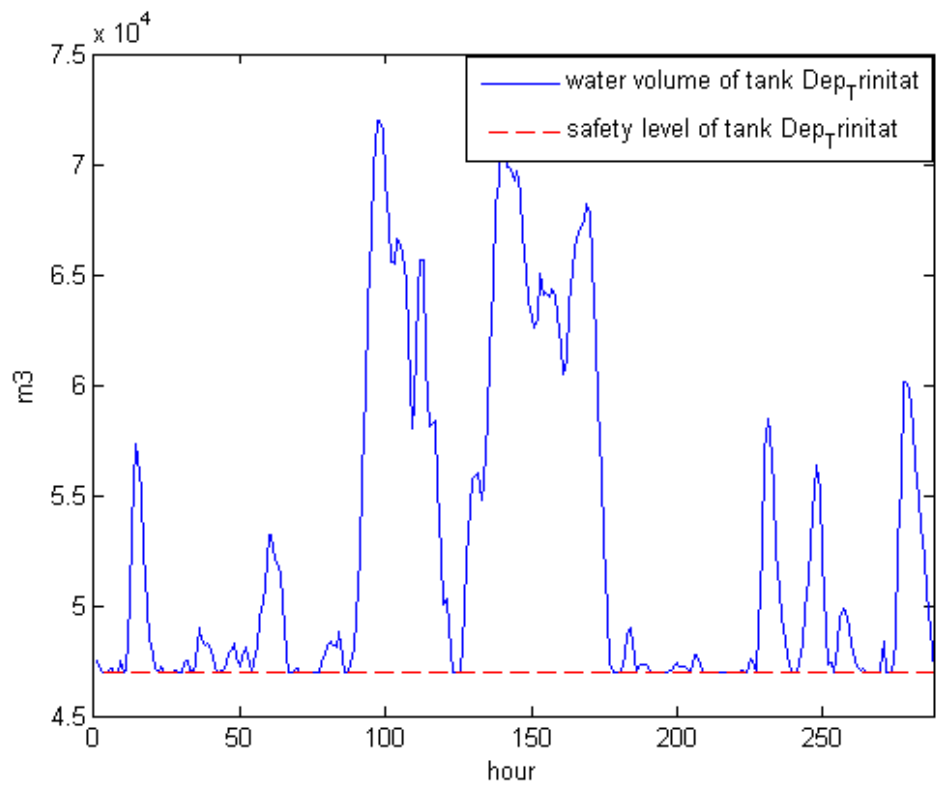

Figure 6: Water of reservoir comparing with safety level 
two rivers achieved by the balanced management, as explained in Goal 3, in the MPC optimizer. In this table, Source means flow into the rivers from external sources, Fixed Demand refers to demands which cannot choose their water source while Variable Demand is the demand which can receive water from more than one river. $B D$, abbreviation of Balanced Demand, is water volume that has been consumed from each of the reservoirs and $P B$, abbreviation of Proportion of Balanced demands, is the proportion of $B D$ for the two reservoirs. $P R$, abbreviation of Proportion of Reservoir capacity, is the proportion of storage capacities of the two reservoirs. The similar values for $P B$ and $P R$ is what the multi-layer scheme seeks to achieve. And SA, abbreviation of Supplying Ability, is water supply ability (in days) of the whole water network before meeting deficit problem in case of no rain and no external water flow into the reservoir. The comparisons prove that, after adding the objective of balance management, the proportion of water usage from both rivers $(58.93 \%$, which is ratio of Llobregat/Ter) is much closer to the proportion of their storage capacities (53.48\%). Moreover, the Catalunya Regional Water Network can supply water 65 days longer than without balance management, which is a good benefit regarding the sustainable usage of water resource in the long term perspective.

\subsection{Result of the integrated Scheme}

In the integrated scheme, simulator and MPC controller keep communicating at every time step. MPC optimizer send control action as set-point to the simulator, after simulating, state variables used as initial value for the next iteration. The computation of control strategies by MPC uses a simplified model of the network dynamics. The use of the combined approach of optimization and simulation contributes to making sure that the effect of more complex dynamics, better repre- 
Table 1: Water usage comparison of Scenarios 3

\begin{tabular}{llllllll}
\hline Sc. & \multicolumn{7}{c}{ MPC optimizer with balancing management } \\
\hline Es. & Source & Fixed Demand & Variable Demand & BD & PB & PR & SA \\
\hline L. & 3008 & 2981 & 724 & 697 & $58.93 \%$ & $53.48 \%$ & 242 Days \\
T. & 3532 & 3518 & 1196 & 1182 & & & \\
\hline Sc. & \multicolumn{7}{c}{ MPC optimizer without balancing management } \\
\hline Es. & Source & Fixed Demand & Variable Demand & BD & PB & PR & SA \\
\hline L. & 3008 & 2981 & 7.6 & -19.4 & $-1.02 \%$ & $53.48 \%$ & 177 Days \\
T. & 3532 & 3518 & 1914 & 1900 & & & \\
\hline
\end{tabular}

sented by the simulation model, may be taken into account. State variables, which represent water volume evolution produced by this integrated scheme should be similar with that provided by the independent MPC controller which means MPC controller without communicating with simulator. As Fig. 7 shows, the solid line is water volume evolution from the integrated scheme, while the dashed line shows the water volume produced by independent MPC controller, which work in a similar way.

Another hand, according to the simulation structure, flow in nodes have to keep balance in every simulating and optimizing iteration. In Fig. 8, the number 1 means demands satisfaction and node flow balance.

Fig. 9 compares the value of each operational goal in the objective function of the integrated and the independent control models. These values do not differ by significant amounts, so that the integrated approach does not significantly increase the operational cost.

Table 2 provides results in detailed numbers and compares the obtained control results in terms of economical and computational performance over four days 


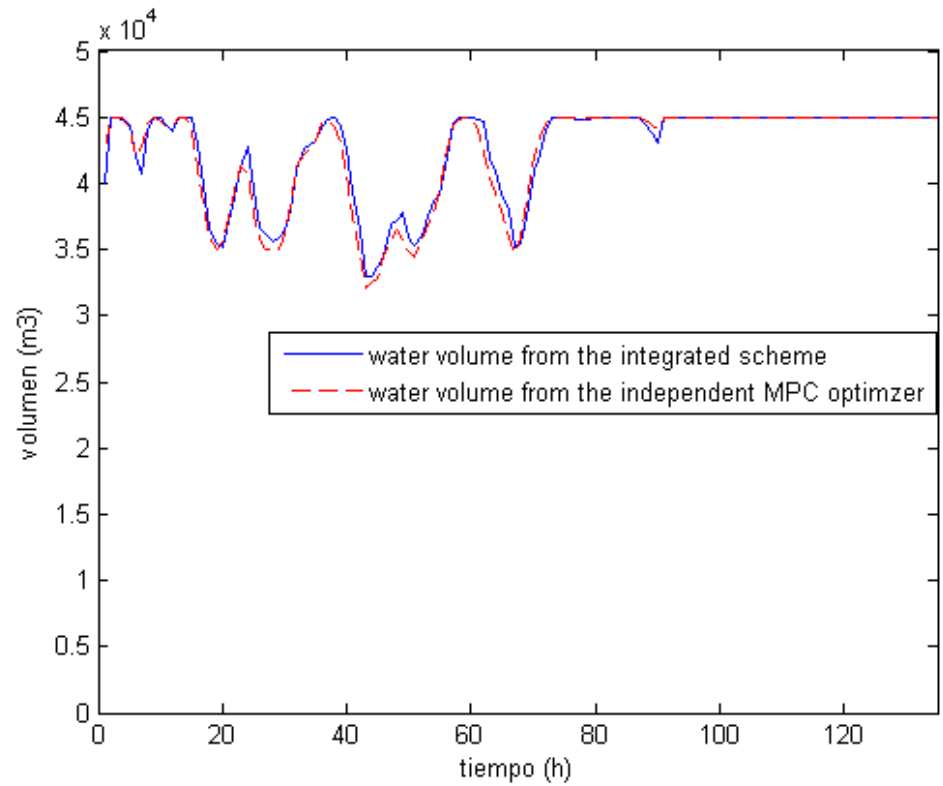

Figure 7: Volume comparison achieved by optimizer and by the integrated scheme
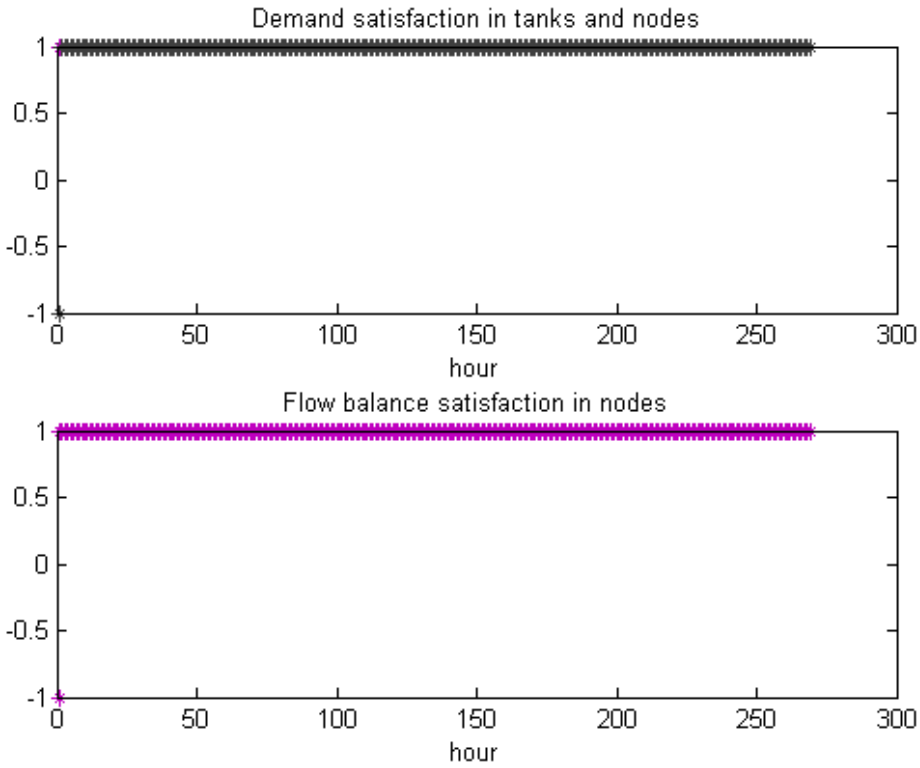

Figure 8: Demands satisfaction and node balance 

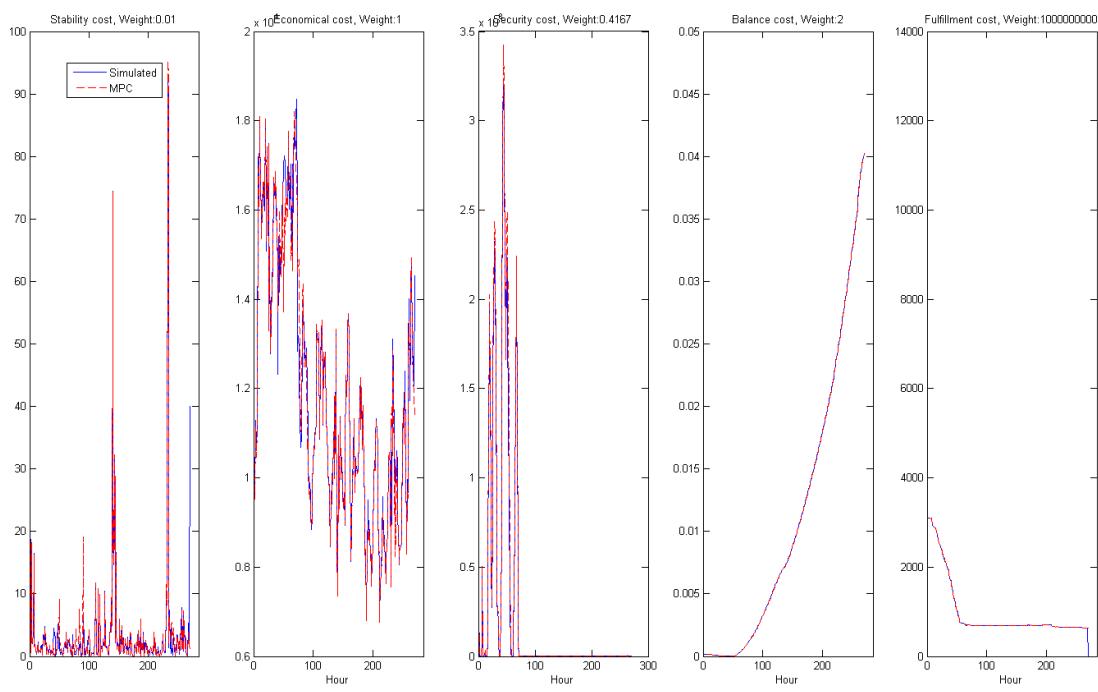

Figure 9: Comparisons of operational goals achieved by optimizer and by integrated scheme

Table 2: Closed-loop performance results (all values in e.u.)

\begin{tabular}{ccccccccc}
\hline \multicolumn{3}{c}{ Define } & \multicolumn{3}{c}{ Current Control } & \multicolumn{3}{c}{ Integrated Scheme } \\
\multicolumn{2}{c}{ Day Ele. } & Wat. & Tot. & Comp.(in s) & Ele. & Wat. & Tot. & Comp.(in s) \\
\hline 1st & 240 & 100 & 340 & 23 & 141 & 40 & 181 & 58 \\
2 nd & 239 & 106 & 345 & 21 & 170 & 39 & 209 & 57 \\
3 3rd & 246 & 94 & 340 & 19 & 171 & 41 & 212 & 61 \\
4 4th & 264 & 110 & 374 & 21 & 168 & 42 & 210 & 62 \\
\hline Prop. & & & & $-34 \%$ & $-61 \%$ & $-42 \%$ & $183 \%$ \\
\hline
\end{tabular}

between the control techniques using heuristic strategies by human operators (simplified as current control) and this integration scheme.

In the Table 2, Wat., abbreviation of Water, refers to water cost during the day, while Ele., abbreviation of Electricity, refers to electricity cost, Tot., abbreviation of Total, means the total cost which includes both water and electricity and Comp., 
abbreviation of Computation time, which means the needed computation time for that optimization process. The column of Proportion is the improved proportion with respect to the current control. In this table, the result shows that the integrated scheme is much better than the current controller at the point of economical cost. On the other hand, computation time is worse because of the size of the water network and also communicating between the two models. In order to solve the computational problem, partitioning or multi-layer integration of simulation and optimization for LSWSS is necessary for the future research.

\section{Conclusions}

This paper presents an integrated simulation and optimization modelling approach which combines the strategic operational control modules with network monitoring in a smooth and synergic way for the real-time LSWSS. This combined approach provides the optimal configuration for LSWSS which is able to optimize and monitor large water systems including reservoirs, open-flow channels for water supply and transport, water treatment plants and so on. MPC, which is the control approach, has been proved the effectiveness of handling complex set of objectives, which can generate flow control strategies from the sources to the consumer areas to meet future demands. Operational goals such as network safety volumes, optimal economic cost, least wasted water, balanced usage of two rivers and flow control stability are represented by a multi-objective function which is optimized by MPC as comparisons of Table 1. Real-time network monitoring is provided by the simulator, which reflects the natural behavior of water flow in a graphically way, and dynamic behaviors of reservoirs in order provide graphical data to the supervisory control and data management system. Comparisons be- 
tween integrated scheme also versify the feasibility of the proposed solution. The case study of Catalunya regional water network has been also emphasize practical meaning of the proposed approach.

\section{References}

[1] Simonovic Slobodan P. Tools for water management: one view of future. Water International, 25:76-88, 2000.

[2] Rani D. and Moreira M.M. Simulation-optimizaiton modeling: A survey and potential application in reservoir systems operation. Water Resource Management, 24(1):1107-1138, 2010.

[3] Miguel Molina. Efficient integrated real-time monitoring and control of drinking water networks. Research proposal, 2011.

[4] Yeh William W-G. Reservoir management and operations models:a stateof-the-art review. Journal of Water Resources and Research, 21:1797-1818, 1985.

[5] Wurbs Ralph A. Reservoir-system simulation and optimization models. Journal of Water Resources Planning and Management, 119:455-472, 1993.

[6] Labadie John W. Optimal operation of multireservoir systems:state-of-theart review. Journal of Water Resources Planning and Management, 130:93$111,2004$.

[7] Wurbs R.A. Modeling and analysis of reservoir system operation. Prentice Hall, Upper Saddle River, 1996. 
[8] ReVelle C. Optimizing reservoir resources:including a new model for reservoir reliability. Wiley, New York, 1999.

[9] McMahon G.F. and Mein R.G. River and reservoir yield. Water Resources Publications, Fort Collins, 1986.

[10] Loucks D.P., Stedinger J.R., and Haith D.A. Water resources systems planning and analysis. Prentice-Hall, Englewood Cliffs, 1981.

[11] Karamouz M., Szidarovszky F., and Zahraie B. Water resources systems analysis. Lewis, Boca Raton, 2003.

[12] Loucks D.P. and Beek V. Water resources systems planning and management: an introduction to method, models and applications. UNESCO, Paris, 2005.

[13] Ocampo-Martinez C., Puig V., Cembrano G., and Quevedo J. Application of predictive control strategies to the management of complex networks in the urban water cycle. IEEE Control Systems Magazine, 33(1):15-45, 2013.

[14] Brdys M.A. and Ulanicki B. Operational Control of Water System. Prentice Hall, London, 1994.

[15] Marinaki M. and Papageorgiou M. Optimal Real-time Control of Sewer Networks. Springer, London, 2005.

[16] Overloop P.J.V. Model Predictive Control on Open Water Systems. Delft University Press, Delft, 2006. 
[17] Negenborn R.R., Schutter B.D., and Hellendoorn J. Multi-agent model predictive control for transportation networks: Serial vs. parallel schemes. Engineering Applications of Artificial Intelligence, 21(3):353-366, 2008.

[18] Bishop Robert H. Modern Control Systems Anaylysis and Design Using MATLAB and SIMULINK. Addison-Wesley Longman Publishing Co., Boston, 1996.

[19] Caini Elena. Sviluppo di un Tool di Simulazione per il Controllo Predittivo di Reti Idriche di Trasporto di Acqua Potabile. PhD thesis, Facolta di ingegneria, Universita Degli Studi Di Siena, 2009.

[20] Mays L. Urban Stormwater Management Tools. McGrawHill Professional Publishing, USA, 2004.

[21] Litrico X. and Fromion V. Modeling and control of hydrosystems. Springer Dordrecht Heidelberg London, New York, 2009.

[22] Cembrano G., Quevedo J., Salamero M., Puig V., Figueras J., and Marti J. Optimal control of urban drainage systems. a case study. Control Engineering Practice, 12(1):1-9, 2004.

[23] Ocampo-Martinez C., Barcelli D., Puig V., and Bemporad A. Hierarchical and decentralised model predictive control of drinking water networks: Application to barcelona case study. IET Control Theory and Applications, 6(1):62-71, 2012.

[24] Litrico X. and Fromion V. Simplified modeling of irrigation canals for controller design. Journal of Irrigation and Drainage Engineering, 130:373383, 2004. 
[25] Quevedo J., Puig V., Cembrano G., and Blanch J. Validation and reconstruction of flow meter data in the barcelona water distribution network. Control Engineering Practice, 11:640-651, 2010.

[26] Maciejowski J.M. Predictive Control with Constraints. Prentice Hall, Great Britain, 2002.

[27] Toro R., Ocampo-Martinez C., Logist F., Van Impe J., and Puig V. Tuning of predictive controllers for drinking water networked systems. Proc. 18th IFAC World Congress, 2011.

[28] Chaturvedi and Srivastave. Study of a complex water-resources system with screening and simulation-models. Journal of Water Resources and Research, 17:783-794, 1981.

[29] Kuo Jan-Tai, Hsu Nien-Sheng, Chu Wen-sen, Wan Shian, and Lin Youn-Jan. Real-time operation of tanshui river reservoirs. Journal of Water Resources Planning and Management, 116:349-361, 1990.

[30] Johnson Sharon A., Stedinger Jery R., and Staschus Konstantin. Heuristic operating policies for reservoir system simulation. Journal of Water Resources and Research, 27:673-685, 1991.

[31] Maass Arthur, Hufschmidt Maynard M., Dorfman Robert, JR Harold A. Thomas, Marglin Stephen A., and Fair Gordon Maskew. Design of water resources systems. Harvard University Press, Cambridge, 1962.

\section{Author biographies}

Congcong Sun received her Bachelor degree of Computer Science and Technology from Nanjing Audit University(NAU) in June of 2008, and later on, obtained 
her master Degree in Systems Engineering from Tongji University(TJU) in March of 2011. She is currently a research fellow from the SAC-ESAII research group at IRI and also temporary academic visitor in Water Center of Exeter University. Her main research interests are Multi-layer MPC, Non-linear control theory and also coordinating management of large scale systems.

Vicenç Puig received the telecommunications engineering degree in 1993 and the Ph.D. in control engineering in 1999, both from the Technical University of Catalonia, Barcelona, Spain. He is currently professor of automatic control and leader of the advanced control systems research group at the Universitat Politècnica de Catalunya. His main research interests are fault detection and isolation, faulttolerant control of dynamic systems, and model predictive control of large-scale systems with special emphasis on water systems. He has been involved in several European projects and networks and has published approximately 200 papers in international conference proceedings and about 45 in scientific journals.

Gabriela Cembrano received her M.Sc. and Ph.D. in industrial engineering and automatic control from the Technical University of Catalonia in 1984 and 1988, respectively. Since 1989, she has been a tenured researcher of the Spanish National Research Council at Instituto de Robótica e Informática Industrial (CSICUPC) and CETaqua Centro Tecnológica del Agua (Agbar-CSIC-UPC). Her main research area is control engineering, and she has been involved in numerous industrial projects on the modeling and optimal control of water supply, distribution, and urban drainage system since 1985. She has taken part in several Spanish and European research projects in the field of advanced control and especially its application in water systems. Currently, she is the leader of the WATMAN project on the modeling and control of systems in the water cycle, funded by the 
Spanish Ministry of Science and Innovation (2010 - 2012) and scientific director of the European Commission project EFFINET Integrated Real-time Monitoring and Control of Drinking Water Networks FP7ICT - 318556(2012 - 2014). She has published over 50 journal and conference papers in this field. 